\title{
Extraction, Purification and Analysis of Sweet Compounds in Stevia rebaudiana Bertoni using Chromatographic Techniques
}

\author{
NEENA KUMARI*, R. C. RANA, Y. P. SHARMAAND S. KUMAR ${ }^{1}$ \\ Department of Forest Products, Dr. Y. S. Parmar University of Horticulture and Forestry, Nauni, Solan-173 230, ${ }^{1}$ Department \\ of Forestry, Mizoram University, Aizawl-796 004, India
}

\section{Neena, et al.: Estimation of Diterpenoid Glycosides of $S$. rebaudiana}

\begin{abstract}
Stevioside and rebaudioside-A are two commercially important diterpenoid glycoside natural sweeteners present in Stevia rebaudiana Bertoni. Stevioside is the major component about 300 times sweeter than sugar, while rebaudioside- $A$ is 1.6 to 1.8 times sweeter than stevioside. Some of the modern extraction and purification techniques developed have been found to be complex and expensive. Therefore, an attempt was made to extract and purify stevioside and rebaudioside- $A$ using simple, easy and inexpensive thinlayer chromatography and column chromatography techniques. Dried leaves were extracted with methanol followed by purification through column chromatography and further analysis was done through high performance liquid chromatography. This extraction method provided a simple and cost effective route to obtain almost pure stevioside and rebaudioside-A.
\end{abstract}

Key words: Stevia rebaudiana, rebaudioside-A, stevioside, column chromatography, thin layer chromatography

Nutrition and health demands are rising continuously and thus expanding the sweetener market. In today's world, there is an increasing demand for natural sweeteners. These are not only low calorie holders but are also health compatible. Stevia based sweeteners have been in use from a long time back in the history. Stevia comprises of 150-300 species, distributed in the New World from the south western United States to the northern Argentina ${ }^{[1,2]}$. Stevia rebaudiana Bertoni is the only known species gifted with a huge potential as a sweetener. Plant is a perennial shrub of the Asteraceae family and native to South America (Paraguay and Brazil). It is known as sweet weed, sweet herb, sweet leaf and honey leaf ${ }^{[3,4]}$. Chemical constituents responsible for sweetness are diterpenoid glycosides and are primarily present in the leaves. Stevioside and rebaudioside-A are the major sweetening compounds (fig. 1). Stevioside has bitter aftertaste while, rebaudioside-A is without bitterness and sweetness is 1.6-1.8 times higher than stevioside ${ }^{[5-7]}$. Stevioside is used in several parts of the world as a low calorie sweetener. In addition to sweetening properties, leaves of $S$. rebaudiana has therapeutic properties like anticariogenic ${ }^{[8]}$, antimicrobial ${ }^{[9]}$,

*Address for correspondence E-mail: neenak.kashyap@gmail.com antiviral $^{[10]}, \quad$ antifungal $^{[11]}, \quad$ antihypertensive ${ }^{[12]}$, antihyperglycaemic ${ }^{[13,14]}$, antitumour ${ }^{[15]}$, antiinflammatory ${ }^{[16]}$, antidiarrheal, diuretic, antihuman rotavirus activities ${ }^{[17]}$, antiHIV ${ }^{[18]}$, hepatoprotective ${ }^{[19]}$ and immunomodulatory ${ }^{[20]}$. Extraction and purification of active chemicals from plant extracts involves various techniques. There were several published literatures on extraction and purification of stevioside and rebaudioside-A using different solvents, solvent plus decolorizing agent, adsorption and ion exchange chromatography, supercritical fluid extraction and ultra and nano membrane filtration ${ }^{[21-24]}$. Supercritical fluid extraction of stevioside from $S$. rebaudiana leaves had been optimized using modifier resulting in improved yield of stevioside as compared to using $\mathrm{CO}_{2}$ at any temperature and pressure. Stevioside yield was found $150 \%$ greater by $\mathrm{CO}_{2}:$ methanol:water $(80: 6: 4)$

This is an open access article distributed under the terms of the Creative Commons Attribution-NonCommercial-ShareAlike 3.0 License, which allows others to remix, tweak, and build upon the work non-commercially, as long as the author is credited and the new creations are licensed under the identical terms

Accepted 30 May 2017

Revised 28 January 2017

Received 29 August 2016

Indian J Pharm Sci 2017;79(4):617-624 


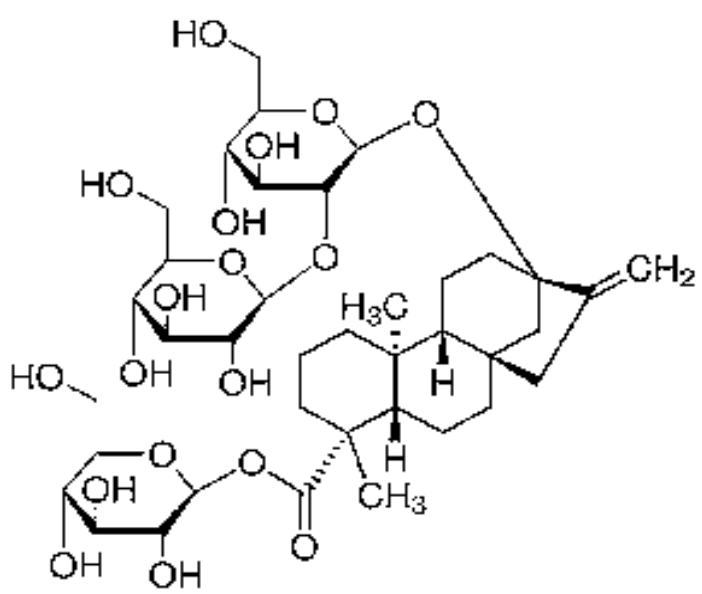

A

Fig. 1: Chemical structure of stevioside and rebaudioside-A A. Stevioside; B. Rebaudioside-A

than conventional organic extraction ${ }^{[25]}$. Modern developed extraction and purification techniques give the high purity stevioside and rebaudioside-A, but are costly and complex too. For instance, microwave assisted extraction (MAE) method for the extraction of stevioside and rebaudioside-A from $S$. rebaudiana leaves was developed. This method yielded 8.64 and 2.34 percent of stevioside and rebaudioside-A, while conventional and ultrasound techniques yielded 6.54 and 1.20 percent and 4.20 and 1.98 percent of stevioside and rebaudioside-A, respectively ${ }^{[26]}$. Later on, stevioside was extracted from $S$. rebaudiana leaves using enzymatic extraction method, which resulted in improved stevioside yield. Hemicellulase was observed to give the highest stevioside yield $(369.23 \pm 0.11 \mu \mathrm{g})$ in $1 \mathrm{~h}$ in comparison to cellulose $(359 \pm 0.30 \mu \mathrm{g})$ and pectinases $(333 \pm 0.55 \mu \mathrm{g})^{[27]}$. So, from economic view point and complexity regarding modern extraction method, mentioned above, the researchers on the small scale are still using conventional techniques for extraction of diterpene glycosides in spite of taking more time. Thus, considering its applicability, commercial importance and above said points, present study was carried out for extraction, purification and analysis of stevioside and rebaudioside-A through using conventional techniques.

\section{MATERIALS AND METHODS}

The chemicals, reagents and solvents used for the extraction of steviol glycosides and carrying out

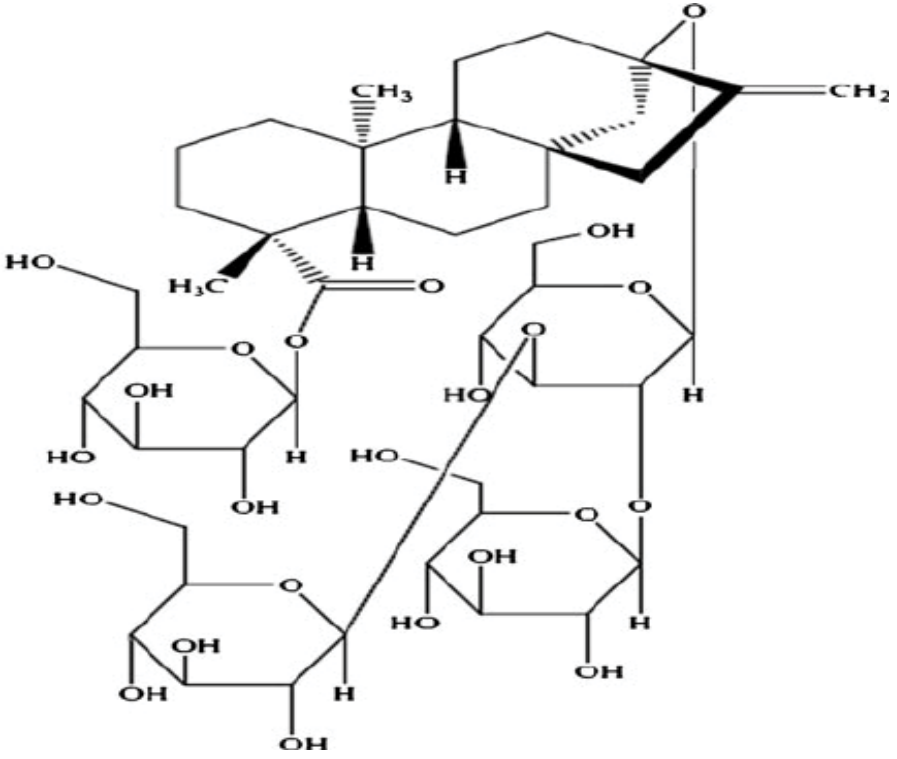

B thin layer chromatography (TLC) and column chromatography were of analytical grade of S.D. FineChem, Thomas Baker and CDH. High performance liquid chromatography (HPLC) was carried out by using HPLC grade solvents. Reference standards of two sweet compounds (stevioside and rebaudioside-A) of ChromaDex were procured from Life Technologies (India) Pvt. Ltd. Extraction of sweet glycosides was carried out by refluxion method. TLC of plant extracts and column chromatography fractions was performed on TLC grade silica gel G and silica gel pre coated aluminium plates of Merck (TLC silica gel $60 \mathrm{~F}_{254}$, $0.2 \mathrm{~mm}$ thickness, $20 \times 20 \mathrm{~cm}$ ). A glass chamber was used for developing TLC plates. TLC plates were developed in two solvent systems, chloroform:methanol:water (65:25:4) and lower layer of chloroform:methanol:water (65:30:10). Spots/compounds on TLC plates were visualized using iodine and $10 \%$ aqueous solution of concentrated sulphuric acid.

The sweet compounds from plant extract was separated through repeated column chromatography on silica gel (60-120 mesh) loaded glass columns using chloroform:methanol (95:5 to 85:15) as the solvent system for elution. Purity assessment of isolated stevioside and rebaudioside-A were performed using Waters HPLC system equipped with binary gradient 515 pump, Rheodyne injector and Waters 2487 dual $\lambda$ absorbance detector. Chromatographic analysis was achieved using Merck Purosphere Star $\mathrm{NH}_{2}$ column $(4.6 \times 250 \mathrm{~mm}, 5 \mu \mathrm{m})$. Acetonitrile:water $(80: 20)$ at 
a flow rate of $1 \mathrm{ml} / \mathrm{min}$ was used as mobile phase to obtain high resolution chromatograms. Ultraviolet detection was set at $210 \mathrm{~nm}$. Millipore filter papers were used for filtering mobile phase solvents (GVWPO $4700,0.22 \mu \mathrm{m}$ ) and plant extracts (GVWPO 1300, 0.22 $\mu \mathrm{m})$. After filtration, the mobile phase solvents were separately degassed on a sonicator and then mixed together.

\section{Plant material:}

Sample plants of $S$. rebaudiana were identified at the Herbarium of Department of Forest Products, Dr. Y. S. Parmar University of Horticulture and Forestry, Nauni Solan (Himachal Pradesh). Leaves were harvested from few $S$. rebaudiana plants grown in the experimental farm of the Department. Leaves were initially dried for $10 \mathrm{~d}$ in the open under shade by spreading on filter paper sheets and then final drying was done in the oven $\left(60^{\circ}, 4 \mathrm{~h}\right)$.

\section{Extraction of sweet compounds:}

The leaves were powdered and extraction of sweet glycosides was carried out with methanol ${ }^{[28,29]}$. Powdered leaf material $(250 \mathrm{~g})$ was refluxed with 21 methanol for $1 \mathrm{~h}$ in a round bottom flask on a hot water bath. The contents were then cooled and filtered under vacuum. The plant residue was further refluxed with methanol three times and each time the filtrates were collected. All the filtrates were combined and solvent was distilled off on a hot water bath. The residue thus obtained was completely dried under vacuum and then it was refluxed $(1 \mathrm{~h})$ with chloroform $(500 \mathrm{ml})$ on a hot water bath. After cooling, the chloroform layer was decanted and collected in a separate flask. The process was repeated till there was no colour in chloroform. The chloroform insoluble part was dissolved in methanol $(250 \mathrm{ml})$ by gentle heating and kept overnight in the refrigerator. The settled mass was filtered and washed with methanol. The main filtrate was combined with methanol washings and distilled on a water bath. The residue thus obtained was vacuum dried and dissolved in distilled water $(400 \mathrm{ml})$ and repeatedly extracted $(200 \mathrm{ml} \times 5)$ with n-butyl alcohol in a separating funne ${ }^{[30]}$. All the n-butyl alcohol layers (upper layers) were combined and solvent was distilled off in a rotavap to give light brown residue. The residue was dissolved in methanol and run on a TLC plate to check the presence of sweet glycosides.

\section{Separation of sweet compounds through column chromatography:}

A part of the residue $(10 \mathrm{~g})$ containing sweet steviol glycosides obtained after distillation of n-butyl alcohol was dissolved in minimum amount of methanol in a beaker and mixed with column chromatography grade silica gel (60-120 mesh, $20 \mathrm{~g}$ ). After complete mixing with spatula, methanol was initially evaporated in the open and finally it was completely dried in a vacuum desiccator. The fine adsorbed mixture was then loaded on a clean and dry glass column packed with silica gel (60-120 mesh) and eluted with chloroform:methanol (95:5 to 85:15). Fractions of $50 \mathrm{ml}$ were collected and after distilling off the solvent, the residues were dissolved in methanol. These fractions were then monitored on silica gel pre-coated TLC plates using lower layer of chloroform:methanol:water $(65: 30: 10)$ as the mobile phases. The spots were visualized by iodine and sulphuric acid (10\%). All the fractions containing similar compounds (appearing as single spots) were combined, concentrated and vacuum dried. The vacuum dried extract was then refluxed with methanol, cooled and allowed to precipitate the stevioside crystals ${ }^{[30]}$. These crystals were separated from the mother liquor through filtration. Mother liquor is heated, concentrated, vacuum dried and treated with a mixture of ethanol and water $(9: 1)^{[31]}$. The solution was heated at a low temperature for $45 \mathrm{~min}$ and then allowed to cool. During this time, precipitates were formed, which were filtered and dried. Repeating the treatment with ethanol-water mixture followed by heating and cooling gives the rebaudioside-A crystals. Authenticity and purity of the isolated compounds were ascertained by running on TLC plates along with reference compounds. Finally their melting points (stevioside-198 ${ }^{\circ}$; rebaudioside-A-243 ${ }^{\circ}$ ) were recorded and matched with the literature values.

\section{Separation of sweet steviol glycoside from partially purified plant extracts:}

The remaining part of residue after extraction with n-butyl alcohol and aqueous extract of leaf material obtained after partial purification were dissolved by gentle heating in minimum volumes of methanol. In each case the contents were cooled and few crystals of pure stevioside were added for initiation of crystallization through seeding and then kept in the refrigerator. After 5-6 d white crystalline stevioside crystallized, which was filtered and washed with methanol. Monitoring on TLC showed stevioside as the major compound and rebaudioside-A as the minor compound along with some other steviol glycosides. 


\section{Analytical method:}

Stock solutions of procured reference compounds (stevioside and rebaudioside-A) were prepared. For preparing stock solutions $25 \mathrm{mg}$ each of stevioside and rebaudioside-A were separately dissolved in $50 \mathrm{ml}$ of acetonitrile:water (80:20) mixture in volumetric flasks giving $500 \mathrm{ppm}$ solutions of each. These were further individually diluted to give solutions of 100, 200, 300 and $400 \mathrm{ppm}$ concentrations for preparing standard curves. Isolated stevioside and rebaudioside-A was then dissolved in acetonitrile:water (80:20) mixture. Before injecting the solutions of reference compounds and isolated stevioside and rebaudioside-A, HPLC column was run in HPLC grade methanol for $3 \mathrm{~h}$ and then with acetonitrile:water (80:20) solvent mixture for $30 \mathrm{~min}$. Flow rate was maintained at $1.0 \mathrm{ml} / \mathrm{min}$. Before using amino column, Waters $\mathrm{C}_{18}$ column was tried for separation of stevioside and rebaudioside-A peaks using methanol:water and acetonitrile:water solvent systems separately in different proportions. However, separation of the two compounds was not possible with none of the above solvent systems on $\mathrm{C}_{18}$ column.

\section{RESULTS AND DISCUSSION}

Sweet compounds enriched extract was obtained by repeated extraction of $S$. rebaudiana leaves with methanol (fig. 2). Methanol extract showed two zones on TLC plates. The methanol extract on complete

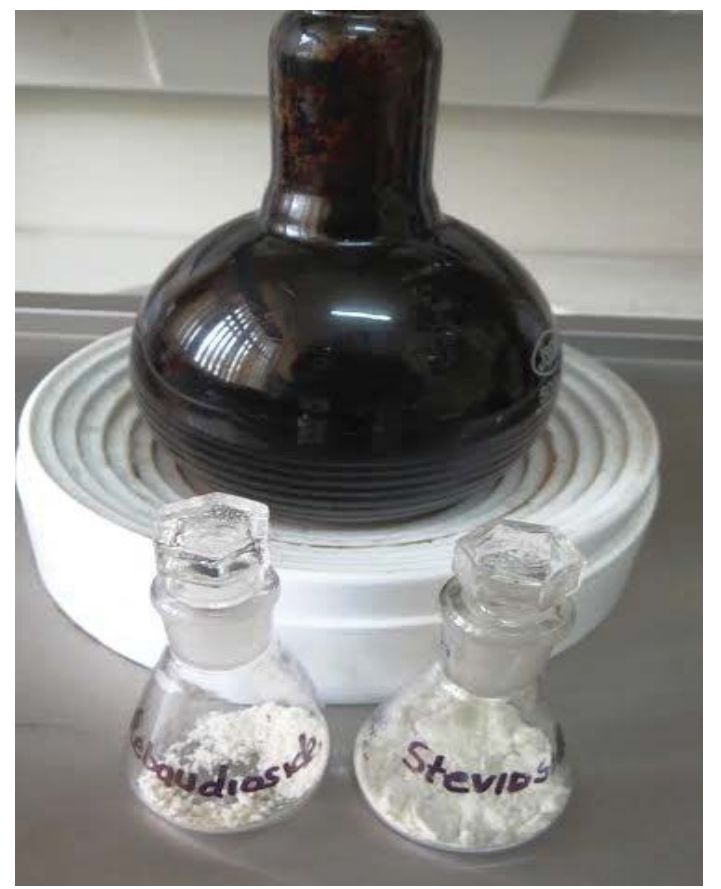

Fig. 2: Leaf extract of Stevia rebaudiana, isolated stevioside and rebaudioside- $A$

July-August 2017 evaporation of solvent followed by vacuum drying and repeated extraction with chloroform resulted in removal of majority of colors and less polar compounds. The absence of sweet compounds was confirmed by running TLC of chloroform extracts. The yield for dried residual extract was $57.50 \mathrm{~g}$. Repeated extraction of aqueous solution of the resultant mass with n-butyl alcohol resulted in separation of steviol glycosides from other most polar water soluble compounds such as oligosaccharides and sweet steviol glycosides were extracted in n-butyl alcohol. Plants contain variety of components ranging from non-polar to polar compounds. It is essential to have thorough knowledge of chemical structures of the compounds to be isolated along with their polarities for selection of the appropriate solvent for their extraction. However, even after using the appropriate solvent with polarity compatible with that of the compounds to be extracted, other compounds also get extracted along with the target compounds. The undesired compounds are required to be removed from the plant material either before extraction of the desired compounds or after their extraction. The sweet compounds present in $S$. rebaudiana belong to diterpenoid type and are glycosidic in nature. These compounds behave polar in nature (due to glycosidic nature) and thus their extraction is carried out with polar solvents like alcohol (methanol/ethanol) or water. During the present studies, the polar sweet diterpenoid glycosides were extracted from the plant material with methanol, however, along with it less polar compounds including colors also got extracted, which were removed by extraction with chloroform from the dried extract. Partitioning of the non-polar compounds free extracts between water and n-butyl alcohol resulted in further purification when sweet diterpenoid glycosides were retained in n-butyl alcohol layer. Methanol was used as extraction solvent at a temperature of $65^{\circ}$ for duration of $1 \mathrm{~h}$, which resulted in complete extraction of stevioside and rebaudioside-A. Methanol, ethanol and water are the commonly used extraction solvents for stevioside and rebaudioside- $\mathrm{A}^{[28,29,32]}$. However, methanol is widely used, as it is easy to distil out due to its lower boiling point $\left(65^{\circ}\right)$. Other reports also suggested methanol as a best solvent at extraction duration of 1 to $1.5 \mathrm{~h}$ in terms of higher stevioside and rebaudioside-A yield ${ }^{[28,33]}$. A drug solvent ratio of 1:8 was used in the present study, which was close to a commonly used 1:10 ratio for extracting bioactive compounds from plants ${ }^{[34,35]}$. Chloroform was used for removal of colours, other 
non-polar compounds and greasy materials from the Stevia leaf extract, as the solvent is non-polar in nature and sweet compounds thus retained behind in the residue were partitioned into butanol from water ${ }^{[28,36-38]}$. Butanol and water are immiscible solvents therefore used advantageously to remove the impurities from the residue, leaving behind the sweet compounds in the butanol layer ${ }^{[39,40]}$. Extraction of $S$. rebaudiana leaves was carried out with water and stevioside isolated through partitioning of sweet enriched residue between water and n-butyl alcohol ${ }^{[39]}$. While, highspeed counter-current chromatography (HSCCC) was applied for isolation of stevioside, rebaudioside-A and rebaudioside-C from leaf extracts of $S$. rebaudiana Bertoni ${ }^{[40]}$. Solvent system (n-hexane:n-butanol:water) at an optimized volume ratio of 1.5:3.5:5, was selected for the HSCCC separation and the lower phase was employed as the mobile phase in the head to tail elution mode. In a single operation, $200 \mathrm{mg}$ of the crude extract yielded pure stevioside (54 mg), rebaudioside -A (36 $\mathrm{mg})$ and rebaudioside- C (13 mg) with the purities of $98.3 \%, 98.5 \%$ and $97.6 \%$, respectively ${ }^{[40]}$.

TLC of methanol extract along with reference compounds showed two distinct spots for stevioside and rebaudioside-A by using chloroform:methanol:water (65:25:4) and lower layer of chloroform:methanol:water (65:30:10) as the two solvent systems and iodine and aqueous solution (10\%) of sulphuric acid as developing reagents. These spots showed black and light brown colors on spraying with sulphuric acid solution (10\%) and keeping in iodine chamber, respectively. The desired resolution of separation was achieved using lower layer of chloroform:methanol:water (65:30:10) as the solvent system. The $\mathrm{R}_{\mathrm{f}}$ value of stevioside and rebaudioside-A (for reference and isolated) were 0.4 and 0.3 , respectively. These results showed that these solvents can be used for separation of compounds in column chromatography.

Residue obtained after distillation of n-butyl alcohol was subjected to column chromatography. The elution was done using chloroform:methanol (95:5 to 85:15) as solvent. Initial fractions showed the presence of colors. Further, on gradually increasing the polarity of the solvents, first stevioside along with some colors started eluting followed by a mixture of stevioside and rebaudioside-A. Fractions showing same pattern in TLC were pooled and concentrated. Methanol addition to the concentrate resulted in the crystallization of stevioside. While, repeated crystallization with methanol resulted in almost pure stevioside (95\%).
However, highly pure rebaudioside-A crystals (98\%) were obtained on concentrating, drying and repeated treatment of mother liquor with ethanol-water mixture. Chloroform:methanol was used as a solvent system starting from a ratio of $95: 5$ and finally to $85: 15$, so that large amount of less polar compounds and colors elutes first with the chloroform in the system. Gradual decrease in the volume of chloroform added to the solvent system resulted in the removal of remaining less polar compounds and colors. Instead, the use of increasing amount of methanol in the system resulted in the complete elution of more polar compounds like, stevioside and rebaudioside-A from the extract. The results were supported by several other workers who used mixture of chloroform and methanol with gradual increasing polarity for purification of steviol glycosides through column chromatography technique ${ }^{[30,41]}$. The various steps involved in extraction and purification of stevioside and rebaudioside-A was given in fig. 3. Separation of pure stevioside crystals were obtained through addition of small amount of methanol to the fractions containing stevioside and rebaudioside-A. These results are in conformity with those reported by other workers ${ }^{[31,42]}$. Former, a method for obtaining highly pure stevioside (99\%) and rebaudioside-A (98\%) from $S$. rebaudiana leaves. $S$. rebaudiana leaves were extracted with water followed by purification with calcium hydroxide and iron chloride. The filtrate obtained was deionized on Amberlite FPC23H, Amberlite FPA51 and Amberlite FPA98Cl. The filtrate was concentrated under vacuum and spray dried. The extract was dissolved in methanol at ambient temperatures to precipitate stevioside. Recrystallization with methanol resulted in pure stevioside. However, concentrating, drying of mother liquor and further treatment with ethanol-water resulted in pure rebaudioside-A formation ${ }^{[31]}$. Later yielded stevioside and rebaudioside-A with purity more than 95 percent through using amberlite XAD-7 resin column. Anhydrous methanol was used for precipitation of the stevioside from a mixture of sweet glycosides. Pure rebaudioside-A crystals were obtained through concentrating and cooling of anhydrous methanol solution ${ }^{[42]}$. Recovery of stevioside from leaves of $S$. rebaudiana by extraction with water as solvent (temperature from room temperature to about $65^{\circ}$ ) with constant stirring and subsequent centrifugation have also been reported. Purification was done by treatment of leaf extract with calcium hydroxide, whereupon a precipitate was obtained by means of filtration or centrifugation. This precipitate was treated with a 


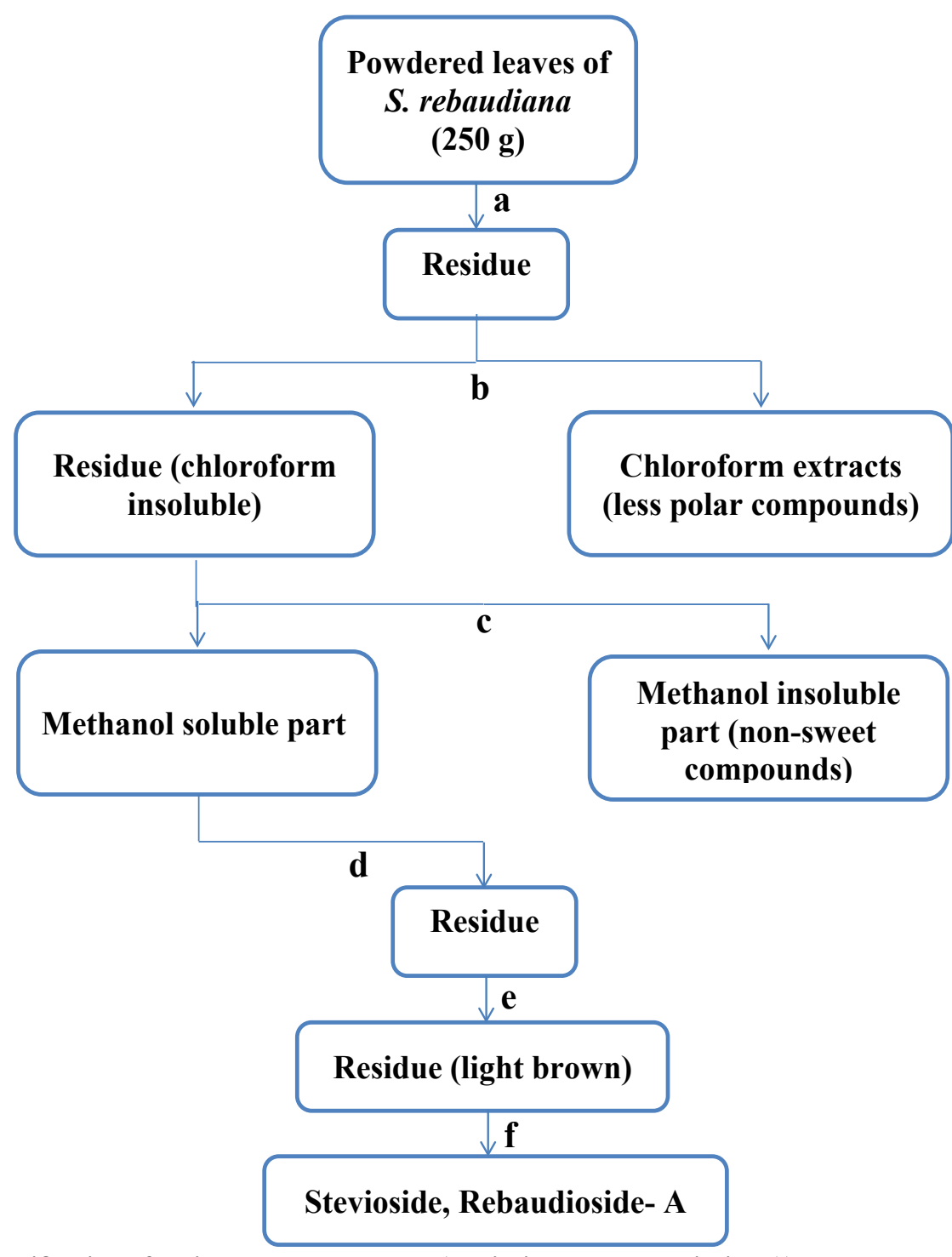

Fig. 3: Extraction and purification of major sweet compounds (stevioside and rebaudioside-A)

(a) Repeatedly extracted $(2 \times 1 \mathrm{~h})$ with methanol through refluxion. Filtered each time, combined the filtrates and distilled off the solvent; (b) refluxion with chloroform; (c) dissolved in minimum volume of methanol and kept overnight in refrigerator. Filtered off insoluble part and washed with methanol; (d) distilled off methanol; (e) added water (200 ml), extracted with n-butyl alcohol $(3 \times 200 \mathrm{ml})$, combined all $\mathrm{n}$ - butyl alcohol layers and distilled off solvent rotavapor; (f) column chromatography (60-120 mesh)

strongly acidic ion exchange resin and subsequently with a weakly basic ion exchange resin, filtered and dried $^{[43]}$.

Five concentrations $(100,200,300,400$ and 500 $\mathrm{ppm}$ ) of stevioside and rebaudioside-A standards were analysed through HPLC and simple linear regression equations were drawn (Table 1). Isolated stevioside and rebaudioside-A, showed distinct peaks at retention times of about 11.24 and $17.54 \mathrm{~min}$, respectively (fig. 4). Better separation of stevioside and rebaudioside-A have also been achieved by using amino column and acetonitrile:water $(80: 20)$ as the solvent system in HPLC ${ }^{[44-46]}$. However, acetonitrile
TABLE 1: SIMPLE LINEAR REGRESSION EQUATIONS FOR STANDARDS

\begin{tabular}{lcc}
\hline & \multicolumn{1}{c}{ Equation } & $\mathbf{R}^{2}$ \\
\hline Stevioside & AUC $=29692.7+5148.01 \mathrm{ppm}$ & 0.997 \\
Rebaudioside-A & AUC $=-12092.4+4107.11 \mathrm{ppm}$ & 0.998 \\
\hline
\end{tabular}

and water as the mobile phase at a flow rate of $1 \mathrm{ml} /$ min and a wavelength of $210 \mathrm{~nm}$ have also been used for quantification of stevioside and rebaudioside- $\mathrm{A}^{[46]}$. In the present investigation, conventional extraction method has been used for extracting diterpene glycosides (stevioside and rebaudioside-A) from $S$. rebaudiana leaves. It is very cheap and simple method and only little experience is required. The 


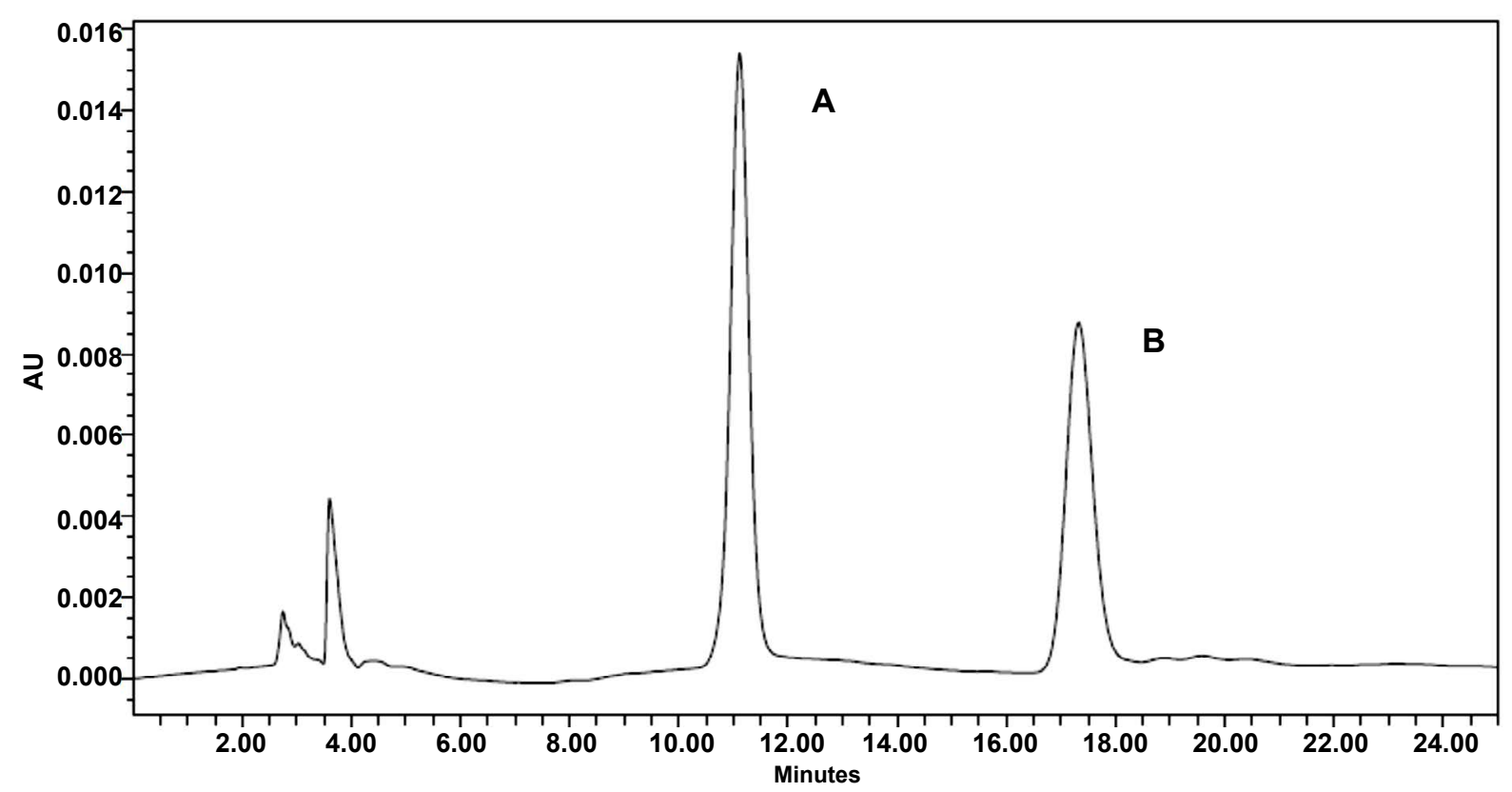

Fig. 4: HPLC chromatogram of isolated stevioside and rebaudioside-A (A) Stevioside; (B) rebaudioside-A

method has the possibility to extract more sample mass than the other methods. Also wide industrial application and better efficiency are advantages of conventional extraction over modern extraction methods. Despite of these advantages, this method has some disadvantages too like it is time consuming and large amount of solvent is required. Stevioside and rebaudioside-A isolated through such studies have significant importance in food and pharmaceutical industries as a low calorie natural sweetener.

\section{Financial assistance:}

None

\section{Conflict of interests:}

None declared

\section{REFERENCES}

1. Amat AG. Los principios edulcorantes de Stevia rebaudiana Bert. Estado actual de sucono cimiento. Acta Farm Bonaer 1982;1:121-23.

2. Soejarto DD, Compadre CM, Medon PJ, Kamath SK, Kinghorn AD. Potential sweetening agents of plant origin II. Field search for sweet-tasting Stevia species. Econ Bot 1983;37:71-79.

3. Liu J, Li SFY. Separation and determination of Stevia sweeteners by capillary electrophoresis and high performance liquid chromatography. J Liq Chromatogr 1995;18:1703-09.

4. Chalapathi MV, Thimmegowda S. Natural non-calorie sweetener stevia (Stevia rebaudiana Bertoni): a future crop of India. Crop Res Hisar 1997;14:347-50.

5. Kaneda A, Kubomura T, Miyazaki K, Wako K, Takahashi C,
Senda S, et al. New sweetener from Stevia. Nikken Chemicals Co. Ltd. Japan Patent No:52-110871;1977.

6. Tanaka O. Steviol glycosides: new natural sweeteners. Trends Anal Chem 1982;1:246-48.

7. Kinghorn AD, Soejarto DD. Current status of stevioside as a sweetening agent for human use. In: Wagner H, Hikino H, Farnsworth NR, editors. Economic and Medicinal Plant Research. New York: Academic Press; 1985. p. 1-52.

8. Gardana C, Scaglianti M, Simonetti P. Evaluation of steviol and its glycosides in Stevia rebaudiana leaves and commercial sweetener by ultra-high performance liquid chromatographymass spectroscopy. J Chromatogr A 2010;1217:1463-70.

9. Satishkumar J, Sarvanan MM, Seethalakshmi I. In vitro antimicrobial and antitumor activities of Stevia rebaudiana (Asteraceae) leaf extracts. Trop J Pharm Res 2008;7:11430-49.

10. Kedik SA, Yartsev EI, Stanishevskaya IE. Antiviral activity of dried extract of Stevia. Pharm Chem J 2009;43:198-99.

11. Silva PA, Oliveira DF, Prado NR, Carvalho DA, Carvalho GA. Evaluation of the antifungal activity by plant extracts against Colletotrichum gloeosporioides PENZ. Ciencia E Agrotecnologia 2008;32:420-28.

12. Hsieh MH, Chan P, Sue YM, Liu JC, Liang TH, Huang TY, et $a l$. Efficacy and tolerability of oral stevioside in patients with mild essential hypertension: a two-year, randomized, placebocontrolled study. Clin Ther 2003;25:2797-808.

13. Jeppesen P, Gregerse S, Gregersen S, Alstrupp K, Hermansen $\mathrm{K}$. Stevioside induces antihyperglycaemic, insulinotropic and glucagonostatic effects in vivo: studies in the diabetic GotoKakizaki (GK) rats. Phytomed 2002;9:9-14.

14. whqlibdoc.who.int/publications/2006/9241660546_eng.pdf.

15. Kaushik R, Narayanan P, Vasudevan V, Muthukumaran G, Antony U. Nutrient composition of cultivated stevia leaves and the influence of polyphenols and plant pigments on sensory and antioxidant properties of leaf extracts. J Food Sci Technol 2010;47:27-33.

16. Arya A, Kumar S, Kasana MS. Anti-inflammatory activity of 
in vitro regenerated calli and in vivo plant of Stevia rebaudiana (Bert.) Bertoni. IJSRP 2012;2:1-5.

17. Takahashi K, Matsuda M, Ohashi K, Taniguchi K, Nakagomi $\mathrm{O}$, Abe Y, et al. Analysis of anti-rotavirus activity of extract from Stevia rebaudiana. Antiviral Res 2001;49:15-24.

18. Takahashi K, Iwata Y, Mori S, Shigeta S. In-vitro anti-HIV activity of extract from Stevia rebaudiana. Antiviral Res 1998;37:A59.

19. Mohan K, Robert J. Hepato protective effects of Stevia rebaudiana Bertoni leaf extract in CCl4-induced liver injury in albino rats. Med Aromat Plant Sci Biotechnol 2009;3:59-61.

20. Chatsudthipong V, Muanprasat C. Stevioside and related compounds: therapeutic benefits beyond sweetness. Pharmacol Ther 2009;121:41-54.

21. Zhang SQ, Kumar A, Kutowy O. Membrane based separation scheme for processing sweeteners from stevia leaves. Food Res Int 2000;33:617-20.

22. Pol J, Varadova Ostra E, Karasek P, Roth M, Benesova K, Kotlarikova $\mathrm{P}$, et al. Comparison of two different solvents employed for pressurised fluid extraction of stevioside from Stevia rebaudiana: methanol versus water. Anal Bioanal Chem 2007;388:1847-57

23. Puri M, Sharma, D, Barrow C J, Tiwary AK. Optimisation of novel method for the extraction of steviosides from Stevia rebaudiana leaves. Food Chem 2012;132:1113-20.

24. Teo CC, Tan SN, Yong JWH, Hew CS, Ong ES. Pressurized hot water extraction (PHWE). J Chromatogr A 2010;121:2484-91.

25. Choi YH, Kim I, Yoon KD. Supercritical fluid extraction and liquid chromatographic-electro spray mass spectrometric analysis of stevioside from Stevia rebaudiana leaves. Chromatogr 2002;55:617-20.

26. Jaitak V, Singh B, Kaul VK. An efficient microwave -assisted extraction process of stevioside and rebaudioside-A from Stevia rebaudiana (Bertoni). Phytochem Anal 2009;20:240-45.

27. Puri M, Sharma D, Barrow CJ, Tiwary AK. Optimization of novel method for the extraction of steviosides from Stevia rebaudiana leaves. Food Chem 2012;132:1113-20.

28. Abou-Arab AE, Abou-Arab AA, Abu-Salem MF. Physicochemical assessment of natural sweeteners steviosides produced from Stevia rebaudiana Bertoni plant. African J Food Sci 2010;4:269-81.

29. Rajab R, Mohankumar C, Murungan K, Harish M, Mohanan PV. Purification and toxicity studies of stevioside from Stevia rebaudiana Bertoni. Toxicol Int 2009;16:49-54.

30. Kaur G, Pandhair V, Cheema GS. Extraction and characterization of steviol glycosides from Stevia rebaudiana Bertoni leaves. J Med Plants Stud 2014;2:41-45.

31. Purkayastha S, Markosyan A, Malsagov M. Process for manufacturing a sweetener and use thereof. U.S. Patent No.: 8337927 B2; 2012.

32. Erkucuk A, Akgun IH, Celiktas YO. Supercritical $\mathrm{CO}_{2}$ extraction of glycosides from Stevia rebaudiana leaves: Identification and optimization. J Supercrit Fluids
2009;51:29-35.

33. Afandi A, Sarijan S, Shaha RK. Optimization of rebaudioside A extraction from Stevia rebaudiana (Bertoni) and quantification by high performance liquid chromatography analysis. J Trop Resour Sustain Sci 2013;1:62-70.

34. Martins PM, Thorat BN, Lanchote AD, Freitas LAP. Green extraction of glycosides from Stevia rebaudiana (Bert.) with low solvent consumption: A desirability approach. ResourceEfficient Technologies 2016;2:247-53.

35. Martins PM, Lanchote AD, Thorat BN, Freitas LAP. Turbo-extraction of glycosides from Stevia rebaudiana using a fractional factorial design. Rev Bras Farmacogn 2017;27:510-18.

36. Kinghorn AD. Overview. In: Kinghorn AD, editor. Stevia: The Genus Stevia. Medicinal and aromatic plants-industrial profiles. Vol. 19. London and NY: Taylor and Francis; 2002. p. 1-17.

37. Kinghorn AD, Nanayakkara NPD, Soejarto DD, Medon PJ, Kamath S. Potential sweetening agents of plant origin: I. Purification of Stevia rebaudiana sweet constituents by droplet counter-current chromatography. J Chromatogr 1982;237:478-83.

38. Rao AB, Reddy GR, Ernala P, Sridhar S, Ravikumar YVL. An improvised process of isolation, purification of steviosides from Stevia rebaudiana Bertoni leaves and its biological activity. Int J Food Sci Technol 2012;47:2554-60.

39. Aminha S, Soumya AN, Govinda RV, Goud BM, Irfath M, Quadri SAP, et al. Isolation and extraction of artificial sweetener (Stevia). World J Pharm Res 2014;3:481-86.

40. Huang XY, Jun Fang Fu, Duo-Long DI. Preparative isolation and purification of steviol glycosides from Stevia rebaudiana Bertoni using high-speed counter-current chromatography. Sep Purific Technol 2010;71:220-24.

41. Talha M, Hussain I, Ullah R, Khan L. Analysis of stevioside in Stevia rebaudiana. J Med Plants Res 2012;6:2216-19.

42. Payzant JD, Laidler JK, Ippolito RM. Method of extracting selected sweet glycosides from the Stevia rebaudiana plant. U.S. Patent No.: 5962678; 1999.

43. Giavanetto RH. Method for the recovery of steviosides from plant raw material. US Patent No.:4,892,938; 1990.

44. Dacome AS, Da Silva CC, Da Costa CEM, Fontana JD, Adelmann J, Da Costa SC, et al. Sweet diterpene glycosides balance of a new cultivar of Stevia rebaudiana (Bert.) Bertoni: isolation and quantitative distribution by chromatographic, spectroscopic, and electrophoretic methods. J Process Biochem 2005;40:3587-94.

45. Kolb N, Herrera JL, Ferreyra DJ, Utiana RF. Analysis of sweet diterpene glycosides from Stevia rebaudiana: improved HPLC method. J Agric Food Chem 2001;49:4538-41.

46. Ozturka EY, Nalbantsoya A, Tagb O, Celiktasa OYA. Comparative study on extraction processes of Stevia rebaudiana leaves with emphasis on antioxidant, cytotoxic and nitric oxide inhibition activities. Ind Crops Prod 2015;77:961-71. 